\title{
Original
}

\section{Colecistectomía laparoscópica ambulatoria. Reporte preliminar de 100 casos}

\author{
Marlen Vega-Vega, Claudio Orlich-Carranza, Karen Gómez-Hernández
}

Resumen

Justificación y objetivo: La laparoscopía es el abordaje de elección para la colecistectomía y tiene grandes beneficios sobre la colecistectomía abierta, que incluyen una mejor recuperación. También se sabe que puede realizarse en forma ambulatoria. Sin embargo, en el sistema de seguridad social costarricense no se ha implementado aún un programa de colecistectomía laparoscópica ambulatoria. El objetivo de este trabajo es demostrar que en Costa Rica, en un hospital clase A, se puede realizar la colecistectomía laparoscópica ambulatoria de una manera segura y de rutina.

Métodos: De febrero a julio de 2005, en el Servicio de Cirugía Uno del Hospital San Juan de Dios, se seleccionaron 100 pacientes de 16 a 61 años, previo consentimiento informado, con colelitiasis, sin comorbilidades de importancia, sin colecistitis ni vesículas escleroatróficas. Los pacientes ingresaron a las 6 de la mañana, se operaron durante las siguientes 4 horas, fueron egresados a las 4 de la tarde y evaluados a los quince días después de la cirugía en la Consulta Externa.

Resultados: Se operaron 100 pacientes, de los cuales 6 requirieron ser hospitalizados. Como única complicación hubo una fístula biliar. No hubo reintervenciones ni mortalidad.

Conclusiones: La colecistectomía laparoscópica es un procedimiento de cirugía mayor que puede realizarse de forma segura y ambulatoria en los hospitales clase A de Costa Rica, si previo a esto existe una adecuada evaluación preoperatoria de los pacientes y un adecuado manejo transoperatorio y postoperatorio.

Descriptores: cirugía ambulatoria, colecistectomía, laparoscópica.

Keywords: ambulatory surgery, cholecystectomy, laparoscopy.

Jefe Servicio Cirugía General Uno. Hospital San Juan de Dios Abreviaturas: CL, colecistectomía laparoscópica; CLA, colecistectomía laparoscópica ambulatoria. Correspondencia: Marlen Vega Vega Email: marvegavega@hotmail.com dravega@gmail.com

ISSN 0001-6002/2006/48/3/119-122 Acta Médica Costarricense, @2006 Colegio de Médicos y Cirujanos
Recibido: 29 de setiembre de 2005

Aceptado: 28 de marzo de 2006

En los últimos 20 años se ha implementado con gran auge la cirugía laparoscópica (CL) como un método de diagnóstico y de tratamiento con ventajas superiores a la cirugía abierta, en la mayoría de los casos ${ }^{~}$. La CL es uno de los procedimientos que más se realiza por esta vía.

En 1985, Erich Mühe realizó la primera colecistectomía cerrada. En 1987, Phillippe Mouret y Francois Dubois la practicaron por primera vez en Francia y son considerados como los pioneros de esta técnica en su país y en el mundo ${ }^{2}$.

La CL tiene varias ventajas, por lo que cada vez se populariza más: menor dolor postoperatorio, menor limitación respiratoria en el periodo postoperatorio, mejor evolución, 
menos estancia postoperatoria, menor tiempo de incapacidad, menor probabilidad de aparición de un ileo paralítico.

Debido a estas ventajas es que en todo el mundo se han implementado programas de cirugía mayor ambulatoria y, específicamente, la colecistectomía como uno de ellos; sin embargo, no está muy difundida en todos los centros como programa ambulatorio. Se empezó a poner en práctica en Estados Unidos a principios de los años $90^{3}$, aunque diversos cirujanos preferían una corta hospitalización de 24 horas. Una experiencia que ha sido utilizada como base para estos programas es la del Brigham and Women's Hospital ${ }^{4}$. Se realizaron 706 CLA, con una tasa de admisión del 3.4\% (25 pacientes). Los siguientes criterios de admisión se aplicaron: ASA I o II, sin antecedentes de reacción adversa a la anestesia, cirugía programada menor de 90 minutos, consentimiento para hacer el procedimiento ambulatorio y viabilidad para ser cuidado por un adulto competente por 24 horas después del procedimiento.

Además, encontraron que hay criterios que predisponen la admisión del paciente, por tanto, no aptos para hacerla ambulatoria: procedimiento de más de 60 minutos, vesícula escleroatrófica o subaguda, adherencias a la vesícula y necesidad de realizar más de un procedimiento.

En múltiples otros centros se han realizado programas similares; en Inglaterra se reporta un estudio con 200 pacientes y otro con $606^{5,6}$. En la Unidad de Cirugía Ambulatoria del Dispensario Central del Ejército en Santa Fe de Bogotá, Colombia ${ }^{6}$, se hizo un programa con 100 pacientes, con una estancia promedio de 7,6 horas. En Chile ${ }^{7}$ se llevó a cabo una experiencia con 357 pacientes en un hospital público, con excelentes resultados y una disminución de la lista de espera. En Zaragoza ${ }^{8}$ se hizo con 265 pacientes y en La Coruña ${ }^{9}$ con 115 , y en un $70 \%$ pudo completarse ambulatoriamente.

En México ${ }^{2,10,11}$ se reportan 2 casuísticas importantes: una en el Hospital General de Puebla ${ }^{10}$, con 10 años de experiencia, en donde se realizaron 405 CLA, de las cuales 306 fueron exitosas. En el Hospital Regional 1 del Instituto Mexicano de Seguro Social en Querétaro ${ }^{2}$ se realizaron 481 CLA, y se concluyó que más del $50 \%$ de los pacientes pueden egresarse el mismo día, en un período de 8 a 10 horas posoperatorias.

En Costa Rica, al momento del presente estudio, no hay un centro que haya reportado la CL ambulatoriamente como un programa regular. En el Hospital San Juan de Dios se inició como un proyecto en febrero de este año, únicamente en el Servicio de Cirugía General Uno. No hay bibliografía nacional que reporte una experiencia como esta.

La tendencia moderna de la cirugía es desarrollar nuevas técnicas y modelos con el apoyo del avance de equipos y materiales, para así disminuir o incluso evitar la hospitalización e incorporar al paciente a sus labores cotidianas lo antes posible.
El creciente aumento de la población costarricense, la disminución del espacio para hospitalizar pacientes, así como disminuir costos en la realización de los programas, fue lo que motivó el inicio de este trabajo.

El objetivo de este estudio es demostrar que puede realizarse la CLA de una manera segura, con una buena evaluación preoperatoria de los pacientes, y un manejo transoperatorio y postoperatorio adecuado.

\section{Materiales y Métodos}

Es un estudio retrospectivo, aprobado y recomendado por el Comité de Ética del Hospital San Juan de Dios, se seleccionaron 100 pacientes del Servicio de Cirugía General Uno del Hospital, que cumplieron con los criterios de selección previamente estipulados para el programa, los cuales fueron una adaptación de los utilizados en la bibliografía consultada ${ }^{4}$ : edad menor de 61 años; sin antecedentes médicos de importancia, curso leve de enfermedades crónicas o inactividad de enfermedad; sin cirugía previa de abdomen superior; residencia a menos de una hora del Hospital; facilidad de transporte de la casa al Hospital; posibilidad de vigilancia por familiar o amigo, por lo menos de un día después del procedimiento; aceptación de ingreso al programa; sin antecedentes de episodios severos de colecistitis o pancreatitis y ultrasonido con vesícula de paredes delgadas.

Los pacientes seleccionados ingresaron el día de la cirugía a las 6 de la mañana, habiendo ayunado por un periodo de 10 horas. Se operaron 2 pacientes cada día, iniciando a las 8 de la mañana. La analgesia postoperatoria consistió de una ampolla de tramadol en $100 \mathrm{ml}$ de suero fisiológico en 30 minutos y metoclopramida ${ }^{2}$ como antiemético (una ampolla en única dosis intravenosa). Los pacientes se egresaron de 8 a 10 horas después del ingreso, con paracetamol en dosis de una tableta cada 6 horas, o ibuprofeno en dosis de una tableta cada 8 horas; la elección del medicamento fue a criterio del cirujano. Los enfermos se controlaron en consulta externa, de 15 a 30 días después de la intervención.

El procedimiento fue llevado a cabo a través de 4 trócares: 2 de 10 milímetros, uno en el ombligo y otro en el epigastrio, 2 de 5 milímetros, que se colocaron en el hipocondrio y el flanco derecho. Se utilizó un lente de 30 grados colocado por el acceso umbilical. Los 2 primeros autores de este artículo realizaron gran parte de los procedimientos, así como los otros cirujanos del Servicio de Cirugía Uno.

Se consideraron como pacientes ambulatorios aquellos que tuvieron una estancia hospitalaria menor a las 12 horas; los que tuvieron una estancia mayor fueron ingresados $\mathrm{y}$ considerados como pacientes hospitalizados. 


\section{Resultados}

Se estudiaron 100 pacientes, que fueron operados del 28 de febrero al 4 de julio de 2005. Dieciocho eran hombres y 82 , mujeres.

Las edades oscilaron entre los 16 y los 61 años. La mayoría de los pacientes se encontraron entre los 34 y los 43 años.

Como antecedentes personales patológicos, 4 tenían enfermedades crónicas previas: artritis reumatoide, hipertensión arterial, intolerancia a los carbohidratos y asma bronquial leve. Se incluyeron estos pacientes cróni$\cos$, ya que la paciente con artritis reumatoide estaba inactiva, la paciente hipertensa se manejaba con un solo medicamento y estaba controlada, la paciente intolerante a los carbohidratos se controlaba muy bien con la dieta y la asmática estaba clasificada como leve (Clasificación GINA) ${ }^{12}$.

Se encontraron antecedentes quirúrgicos presentes en 52 pacientes: 42 con cirugías en hemiabdomen inferior y 9 con cirugías no abdominales. Los otros 48 pacientes no habían sido operados.

Ochenta y seis procedimientos fueron realizados por especialistas y 14 lo fueron por residentes de segundo y tercer año de cirugía general.

El tiempo quirúrgico osciló entre los 15 y los 90 minutos. En 53 pacientes el tiempo operatorio osciló entre 15 y 30 minutos. En 28 pacientes el tiempo operatorio fue de 30 a 45 minutos; en 11, de 45 a 60; en 8 , de 60 a 90 minutos.

En el $94 \%$ de los casos se pudo realizar el procedimiento ambulatorio, mientras que en el $6 \%$ hubo necesidad de admisión (Gráfico 1).

De los pacientes hospitalizados ( 3 mujeres y 3 hombres), 4 se ingresaron por tener vesículas con datos de inflamación subaguda (2 hombres y 2 mujeres), un hombre fue

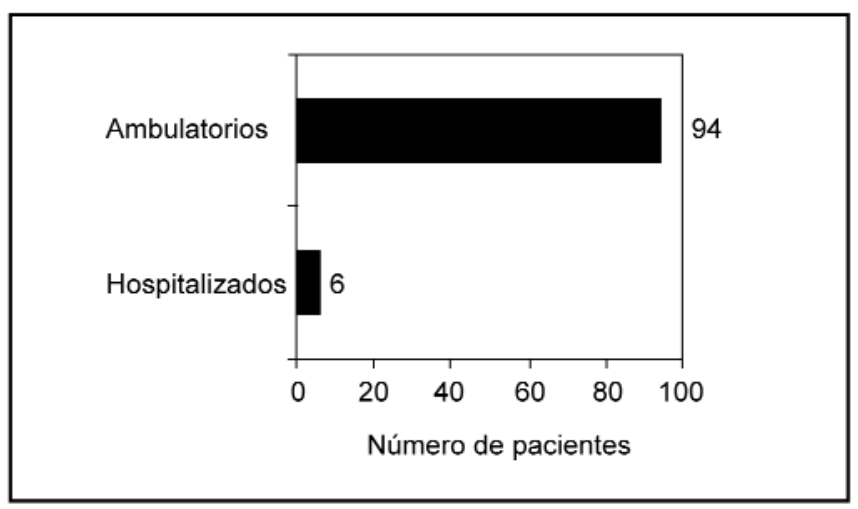

Figura 1. Categoría de admisión. Hospital San Juan de Dios, Cirugía 1, Julio 2005. admitido por tener náuseas postoperatorias y una mujer se dejó para observación, por sangrado transoperatorio.

Hubo una complicación: el caso de una mujer con una colecistitis subaguda, quien desarrolló una fístula biliar; fue una de las pacientes que se dejó hospitalizada por dificultad del procedimiento. La fístula fue manejada conservadoramente y no hubo necesidad de reintervención. El cuadro resolvió a los 4 días de internamiento; se egresó y se dio de alta de Consulta Externa.

Un paciente fue reingresado a través del servicio de Emergencias en el postoperatorio \# 8, con dolor abdominal leve y aumento de la bilirrubina indirecta; no se documentó ninguna anormalidad y fue egresado bien. No hubo necesidad de conversión de CL a abierta.

\section{Discusión}

El integrar una patología quirúrgica a modelos de cirugía ambulatoria supone un período de adaptación, tanto para el cirujano como para su entorno, hasta que llegue a demostrarse que el procedimiento no conduce a un aumento de la morbimortalidad.

La clave del éxito para este tipo de procedimiento y para cualquiera ambulatorio es la información, ya que permite que el procedimiento sea aceptado por parte del paciente y de la familia. Es fundamental informarlos para que comprendan que van a regresar a la casa el mismo día de la cirugía. Además, deben ser informados acerca de los datos de alarma, por los que sería fundamental que consultaran al Servicio de Emergencias.

A nivel mundial se han llevado a cabo ${ }^{2-11}$ varios programas de CL ambulatoria, y en todos se concluye que pueden realizarse con éxito y que la clave es la educación y la información al paciente, la rigurosa selección de los pacientes, los cirujanos expertos, la buena técnica anestésico - analgésica y la existencia de una unidad con una infraestructura adecuada.

En este programa de 100 pacientes se tomaron en cuenta todas las recomendaciones internacionales y se pudieron reproducir en mucho los resultados de las grandes series. Un $94 \%$ de los pacientes terminaron de una manera ambulatoria y un $6 \%$ fueron ingresados. No se presentaron conversiones a cirugía abierta y únicamente hubo una complicación de fístula biliar que se resolvió con manejo conservador; este caso no fue egresado, ya que fue un procedimiento complejo y de más tiempo quirúrgico. No hubo reintervenciones. Se corroboró que un procedimiento de más de 60 minutos tiene alta probabilidad de ingreso ${ }^{5,6,8}$; los pacientes ingresados tuvieron un tiempo quirúrgico mayor de 60 minutos. 
Es ideal tener un ultrasonido reciente al momento del ingreso del paciente, ya que si presenta criterios de inflamación aguda o crónica y el ultrasonido muestra paredes gruesas, el paciente debe excluirse del programa ambulatorio, debido a que el riesgo de ingreso es alto. En nuestra experiencia, 4 de los pacientes se ingresaron por esta razón.

El egreso el mismo día de la cirugía permite una reducción de los costos ${ }^{5,13-15}$ al no existir hospitalización, como se describe en varios estudios. Además, permite que el paciente se incorpore de inmediato al núcleo familiar, lo que genera menos angustia y mayor tranquilidad para la recuperación.

La CLA es un procedimiento de cirugía mayor que debe incluirse como rutina en el programa de cirugía ambulatoria, si se tiene el equipo técnico y humano capacitado para llevar a cabo el procedimiento ${ }^{16,17}$.

El éxito de la CLA reside en la elección adecuada de los pacientes, la buena técnica operatoria, la adecuada anestesia y un tiempo quirúrgico menor de 60 minutos.

La implementación de programas de cirugía ambulatoria es de crucial importancia para hospitales que, como el Hospital San Juan de Dios, tienen una gran demanda de servicios.

\section{Abstract}

Background: It is well known that a laparoscopic approach is the election of choice for cholecystectomies, this is due, among other things, to the great benefits that it has over open surgery regarding patients recovery. It is also known that to do so in an ambulatory setting is reasonable in a subgroup of selected patients. Even though this has become a common practice in other countries, Costa Rica's social security system does not have a program for ambulatory cholecystectomy.

Aim: To demonstrate that in Costa Rica, laparoscopic cholecistectomy can be done safely in an ambulatory setting.

Methods: Between february and july of this year a total of 100 patients with cholelithiasis, with ages between 16 and 61 and a previous ly signed informed consent, were included in a program of ambulatory laparoscopic cholecystectomy at the Surgery One Service at the Hospital San Juan de Dios. In general, these patients had no previous comorbilities. There were no patients with cholecystitis or thicken gallbladder walls on ultrasonography. The patients were admitted at $6 \mathrm{am}$ and had the procedure done some time during the next 4 hours, they were discharged at $4 \mathrm{pm}$ and seen 15 days later.
Results: Of the 100 patients, 6 had to be hospitalized. The only complication was a biliary fistula. There were no reinterventions and there was no mortality.

Conclusions: In Costa Rica, laparoscopic cholecystectomy can be done safely in an ambulatory setting, with an adecuate preoperative selection of patients and an adequate postoperative management.

\section{Referencias}

1. Vega M, Orlich C, Elizondo A. “Extracción laparoscópica de cuerpos extraños intraabdominales“. Gaceta Médica. 2005;7:30-32.

2. Pérez E, Ostos L, Mejía A, García M. “Colecistectomía laparoscópica ambulatoria“. Rev Med IMSS. 2002;40:71-75.

3. Tuckey J, Morris G, Peden C, Tate J. "Feasibility of day case laparoscopic cholecystectomy in unselected patients." Anaesthesia. 1996;24:965-968

4. Lau H, Brooks D. "Predictive Factors for Unanticipated Admissions After Ambulatory Laparoscopic Cholecystectomy“. Arch Surg. 2001;136:1150-1153.

5. Mjaland o, Raeder J, Aasboe V, Trondsen E, Buanes T. “Outpatient laparoscopic cholecystectomy. Br J of Surg. 1997;84: 958-961, 1997.

6. Planells M, García R, Galeano J, López C, Anaya P, Ballester C. et al "Factors predicting patients unit for laparoscopic cholecystectomy on an ambulatory basis“. Br J Surg. 1997;84, Suppl. 2.

7. Zuluaga L, Clavijo W, Villalba A. “Colecistectomía laparoscópica ambulatoria en una Unidad Quirúrgica no hospitalaria“. Rev Colombiana Cir. 2000;15:350-359.

8. Pattillo J, Kusanovic R, Salas P, Reyes J, García I, Sanhueza M. et al "Colecistectomía laparoscópica ambulatoria: una experiencia factible en un hospital público chileno“. Rev MédChile. 2004;132:429-436.

9. Fatás J, Blanco J, Ara J, Dobón M. “Criterios para la realización de colecistectomía laparoscópica ambulatoria dentro de un programa de Cirugía Mayor Ambulatoria“. Revista Asecha. 2000; 5:25-28.

10. Bermúdez I, López S, Sanmillán A, González C, Baamonde I, Rodríguez A, et al. "Colecistectomía laparoscópica en régimen ambularorio". Cir Esp. 2004; 76: 159-163.

11. Justo J, Prado E, Theurel G, De la Rosa R, Lozano A. "Colecistectomía laparoscópica ambulatoria. Una buena alternativa". Cir Gen. 2004; 26: 306-310.

12. National Asthma and Prevention Program. Expert panel report: Guidelines for the diagnosis and management of asthma. National Institutes of Health Publication No. 02-5074 June 2003.

13. Berggren, U., Zethraeus, N., Arvidsson, D., Haglund, U., Jonsson, B. *A cost minimization analysis of laparoscopic cholecystectomy versus open cholecystectomy“. Am J Surg. 1996; 172:305-310.

14. Keulemans Y, Eshuis J, De Haes H. "Laparoscopic cholecystectomy: Day - care versus clinical observation“. Ann Surg 1998; 228:734740.

15. Fleisher S, Yee K, Lillernoe K, Talami M, Yeo C. '"Is outpatient laparoscopic cholecystectomy safe and cost-effective: A model to study transition of care". Anesthesiology 1999; 90:1746-1755.

16. Serra A, Roig M, Lledo J, Santafe A, Espinosa R, Bertomeu C. "The learning curve in Ambulatory Laparoscopic Cholecystectomy”. Surg Laparosc Endosc Percutan Tech 2002;12: 320-324.

17. Rosen M, Malm J, Tarnoff M, Zuccala K, Ponsky J. " Cost Effectiveness of Ambulatory Laparoscopic Cholecystectomy“. Surg Laparosc Endosc Percutan Tech 2001;11: 182-184. 